\title{
ASYMPTOTIC EQUIVALENCE AND SUMMABILITY
}

\author{
MOUSA S. MAROUF
}

Shawner State Iniversily

9.10 2nd Strect

Portsmouth, Oliio $4560^{\circ}$

(Received February 6, 1992 and in revised form June 3, 1992)

ABS'IR $A$ ("I. 'This paper is a study of the relationships between the asymplotic equivalence of two

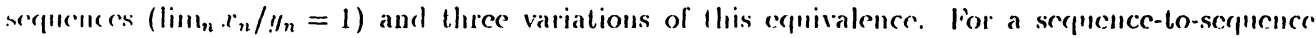

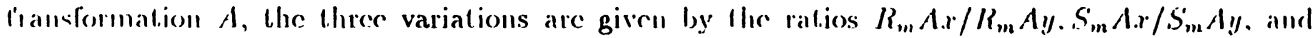
$\mid{ }_{m} A A_{1} / \mu_{m} d !$, where $R_{m} A z:=\sum_{n \geq m}\left|(A z)_{n}\right|, S_{m} A z:=\sum_{n \leq m}\left|(A z)_{n}\right|$, and $\mu_{m} A z:=\sup _{n \geq m}\left|(A z)_{n}\right|$.

KFY WORIS AND PIIRASES. Asymptotically regular. Asymptotic equiralence, Nürland-type matrix $\tilde{N}_{r}$.

1992 ANIS CLASSIFICATION CODES. Primary 40C:05.

\section{INTIROIDUC'TION.}

III [2] Pobyvancts introduced the concept of as.symplotically regular matrices, which preserve the asymptotic equivalence of two nonncgative number sequences; that is, if the quotient $x_{n} / y_{n}$ has limit 1 thon the quotiont $(A x)_{n} /(A y)_{n}$ of the transformed sequences also has a limit 1. Stinterl srmbolically this is $x \sim y$ implies $A x \sim A y$. The frefuent occurrence of terms having \%ero value makes a term-by-term ratio $x_{n} / y_{n}$ inapplicable in many cases. Therefore in [1] Fridy introduced new "lay's of comparing rates of convergence. For $x$ in $\ell^{1}$ he used the remainder sum, whose $m$-th tcrm is $l_{m}, r:=\sum_{n=m}^{\infty}\left|x_{n}\right|$, and cxamined the ralio $R_{m} x / R_{m} y$ an $m \rightarrow \infty$. l'or the case where $x$ is not in $C^{\prime}$ hr used the sequence of partial sums of moduli which is giren by $S_{m} x:=\sum_{n \leq m}\left|x_{n}\right|$. If $x$ is a bounded serfuence he used the supremum of the remaining terms which is given by $\mu_{n} t:=\sup _{n \geq m}\left|t_{n}\right|$. In "acl, case the ratio $x_{n} / y_{n}$ can be replaced by the appropriate new ratio: $R_{m} x / R_{m} y, S_{m} x / S_{m} y$, or $\mu_{m} \cdot x / \mu_{m} . y$. The goal of the work in [1] was to study the relationship of these ratios when they tend to zcro. In the present study we investigate the relationships of these ratios when they have limit onc.

\section{B3.SIT: 'THEOREMS.}

The following results are stated here for the convenience of the reader and to illustrate our notation.

TIIl:ORLiN 1. (Pobyvancl: [2]). The nonnegative matrix $A$ is asymptotically regular if and only if for each fixed integer $m, \lim _{n \rightarrow \infty} a_{n m} / \sum_{k=1}^{x} a_{n k}=0$.

TIII:OREM 2. The Nörland type natrix $\bar{N}_{p}$ is regular if and only if $\lim _{n} P_{n}=\infty$.

IIIlOOREM 3. The matrix $A$ is a $c_{0}-c_{0}$ matrix (i.e.. A preserves zero limits) if and only if

(a) $\lim _{n} a_{n k}=0$ for $k=1,2 \ldots$, and

(b) There exists a number $M>0$ such that for each $n, \sum_{k=1}^{\infty}\left|a_{n k}\right|<M$.

3. AS'YMPTOTIC EQUIVALENCE.

In order to give the main results we first introduce some notalion. 


\section{NOTATIONS:}

$P_{\delta}=\left\{\right.$ the set of all real number sequences such that $x_{k} \geq \delta>0$ for all $\left.k\right\}$

$P_{0}=\{$ the set of all nonnegative sequences which have at most a finite number of zero terms

$P=\left\{\right.$ the set of all sequences $x$ such that $x_{k}>0$ for all $\left.k\right\}$

$$
\bar{N}_{p} \text { is defined by } \bar{N}_{p}[n, k]:=\left\{\begin{array}{lll}
\frac{p_{k}}{P_{n}}, & \text { if } & k \leq n, \\
0 & , \text { if } & k>n,
\end{array}\right.
$$

where $\left\{p_{n}\right\}_{n=0}^{\infty}$ is the sequence of nonnegative real numbers with $p_{0}>0$ and $P_{n}:=\sum_{k=0}^{n} p_{k}$.

THEOREM 1. Let $A$ be a nonnegative $c_{0}-c_{0}$ matrix, and let $x$ and $y$ be bounded sequences such that $x \sim y$ and $x, y \in P_{\delta}$ for some $\delta>0$, then $\mu A x \sim \mu A y$.

PROOF. Since $x \sim y$ we can write $x_{n}=y_{n}\left(1+z_{n}\right)$ for some null sequence $z$. For each $n$ we have

$$
\begin{aligned}
\frac{(\mu A x)_{n}}{(\mu A y)_{n}} & =\frac{\sup _{k \geq n}(A x)_{k}}{\sup _{k \geq n}(A y)_{k}}=\frac{\sup _{k \geq n} \sum_{i=0}^{\infty} a_{k i} x_{i}}{\sup _{k \geq n} \sum_{i=0}^{\infty} a_{k i} y_{i}} \\
& =\frac{\sup _{k \geq n} \sum_{i=0}^{\infty} a_{k i}\left(y_{i}+y_{i} z_{i}\right)}{\sup _{k \geq n} \sum_{i=0}^{\infty} a_{k i} y_{i}} \leq 1+\frac{\sup _{k \geq n} \sum_{i=0}^{\infty} a_{k i} y_{i}\left|z_{i}\right|}{\sup _{k \geq n} \sum_{i=0}^{\infty} a_{k i} y_{i}} \\
& \leq 1+\frac{\sup _{k \geq n} \sum_{i=0}^{\infty} a_{k i} y_{i}\left|z_{i}\right|}{\delta \sup _{k \geq n} \sum_{i=0}^{\infty} a_{k i}} .
\end{aligned}
$$

Since $y$ is a positive bounded sequence, $z$ is a null sequence, and $A$ is a $c_{0}-c_{0}$ matrix, it follows that $\lim _{n} \sum_{i=0}^{\infty} a_{k i} y_{i}\left|z_{z}\right|=0$, and therefore $\lim _{n}\left((\mu A x)_{n} /(\mu A y)_{n}\right) \leq 1$. In a similar manner wc can get. $\lim _{n}\left\{(\mu A x)_{n} /(\mu A y)_{n}\right\} \geq 1$, hence $\lim _{n}\left\{(\mu A x)_{n} /(\mu A y)_{n}\right\}=1$, i.e, $\mu A x \sim \mu A y$.

THEOREM 2. If $A$ is a nonnrgative $\ell^{\infty}-\ell^{1}$ summability matrix then the following are equivalent:

(i) if $x$ and $y$ are bounded sequences such that $x \sim y$ and $y \in P_{\delta}$ for sone $\delta>0$, then $R A x \sim R A y$;

(ii) for each $m$,

$$
\lim _{n \rightarrow \infty}\left(\frac{\sum_{k=n}^{\infty} a_{k m}}{\sum_{k=n}^{\infty} \sum_{j=0}^{\infty} a_{k j}}\right)=0 .
$$

PROOF. [(ii) $\Rightarrow$ (i)] The hypothesis $x \sim y$ implies that for $\varepsilon>0$ there exists a number $J$ such that if $k \geq J$ then $\left|\left(x_{k} / y_{k}\right)-1\right|<\varepsilon$. Hence, for all $k \geq J$ we have

$$
(1-\varepsilon) y_{k} \leq x_{k} \leq(1+\varepsilon) y_{k} \text {. }
$$

First,

$$
R_{n} A x=\sum_{k=n}^{\infty}(A x)_{k} \leq \sum_{k=n}^{\infty} \sum_{j=0}^{J-1} a_{k j} x_{j}+\sum_{k=n}^{\infty} \sum_{j=J}^{\infty} a_{k j} x_{j} .
$$

By using (3.1) we have

$$
R_{n} A x \leq\left(\sum_{j=0}^{J-1} x_{j}\right) \sum_{k=n}^{\infty}\left(\max _{0 \leq j \leq J-1} a_{k j}\right)+(1+\varepsilon) \sum_{k=n}^{\infty} \sum_{j=0}^{\infty} a_{k j} y_{j}
$$


Hence,

Now we claim that

$$
\frac{R_{n} A x}{R_{n} A y} \leq \frac{\left(\sum_{j=0}^{J-1} x_{j}\right) \sum_{k=n}^{\infty}\left(\max _{0 \leq j \leq J-1} a_{k j}\right)}{\delta \sum_{k=n}^{\infty} \sum_{j=0}^{\infty} a_{k j}}+1+\varepsilon
$$

Using (ii), we get

$$
\lim _{n \rightarrow \infty}\left[\frac{\left(\sum_{k=n}^{\infty}\left(\max _{0 \leq j \leq J-1} a_{k j}\right)\right)}{\sum_{k=n}^{\infty} \sum_{j=0}^{\infty} a_{k j}}\right]=0 .
$$

where $J$ is finite; hence,

$$
\sum_{j=0}^{J-1}\left(\frac{\left(\sum_{k=n}^{\infty} a_{k j}\right)}{\sum_{k=n}^{\infty} \sum_{j=0}^{\infty} a_{k j}}\right)=0
$$

$$
0 \geq \lim _{n \rightarrow \infty}\left[\frac{\left(\sum_{k=n}^{\infty}\left(\max _{0 \leq j \leq J-1} a_{k j}\right)\right)}{\sum_{k=n}^{\infty} \sum_{j=0}^{\infty} a_{k j}}\right] .
$$

Since $A$ is a nonnegative matrix we have proved the claim, and it follows that

$$
\lim \sup _{n}\left(\frac{R_{n} A x}{R_{n} A y}\right) \leq 1+\varepsilon .
$$

Second, we seek a lower bound for $R_{n} A x / R_{n} A y$. Consider

$$
R_{n} A x=\sum_{k=n}^{\infty} \sum_{j=0}^{\infty} a_{k j} x_{j} \geq \sum_{k=n}^{\infty}\left(\min _{0 \leq j \leq J-1} a_{k j}\right) \sum_{j=0}^{J-1} x_{j}+\sum_{k=n}^{\infty} \sum_{j=J}^{\infty} a_{k j} x_{j}
$$

Using Inequality (3.1) again we get

$$
R_{n} A x \geq\left(\sum_{j=0}^{J-1} x_{j}\right) \sum_{k=n}^{\infty}\left(\min _{0 \leq j \leq J-1} a_{k j}\right)+(1-\varepsilon) \sum_{k=n}^{\infty} \sum_{j=0}^{\infty} a_{k j} y_{j}-(1-\varepsilon) \sum_{k=n}^{\infty} \sum_{j=n}^{J-1} a_{k j} y_{j}
$$

Therefore

$$
\begin{aligned}
\frac{R_{n} A x}{R_{n} A y} \geq & \left(\frac{\sum_{j=0}^{J-1} x_{j}}{\sup _{j} y_{j}}\right)\left(\frac{\sum_{k=n}^{\infty} \min _{0 \leq J \leq-1} a_{k j}}{\sum_{k=n}^{\infty} \sum_{j=0}^{\infty} a_{k j}}-+(1-\varepsilon)\right. \\
& -\left(\frac{(1-\varepsilon) \sum_{j=0}^{J-1} y_{j}}{\delta}\right)\left(\frac{\sum_{k=n}^{\infty} \max _{0 \leq J \leq J-1} a_{k j}}{\sum_{k=n}^{\infty} \sum_{j=0}^{\infty} a_{k j}}\right) .
\end{aligned}
$$

Using the previous claim we have

$$
\liminf _{n}\left(\frac{R_{n} A x}{R_{n} A y}\right) \geq 1-\epsilon
$$

From (3.2) and (3.3) we conclude that $\lim _{n}\left(R_{n} A x / R_{n} A y\right)=1$, whence $R A x \sim R A y$. 
To show that (i) implies (ii) we choose a fixed positive integer $m$ and define $x$ and $y$ as follows: $y_{k}:=1$ for all $k$ and

$$
x_{k}:= \begin{cases}0, & \text { if } k \leq m, \\ 1, & \text { if } k>m\end{cases}
$$

It is obvious that the conditions in (i) are satisfied by the sequences $x$ and $y$. Consider

$$
R_{n} A x=\sum_{k=n}^{\infty} \sum_{j=0}^{\infty} a_{k j} x,=\sum_{k=n}^{\infty} \sum_{j=m+1}^{\infty} a_{k j}=\sum_{k=n}^{\infty} \sum_{j=0}^{\infty} a_{k j}-\sum_{k=n}^{\infty} \sum_{j=0}^{m} a_{k j}
$$

Thus

$$
\left(\frac{R_{n} A x}{R_{n} A y}\right)=1-\left[\frac{\left(\sum_{k=n}^{\infty} \sum_{j=0}^{\infty} a_{k j}\right)}{\sum_{k=n}^{\infty} \sum_{j=0}^{\infty} a_{k} y_{i}}\right] .
$$

The hypothesis that $A$ is a nonnegative matrix implies

$$
\left(\frac{R_{n} A x}{R_{n} A y}\right) \leq 1-\left[\frac{\left(\sum_{k=n}^{\infty} a_{k m}\right)}{\sum_{k=n}^{\infty} \sum_{j=0}^{\infty} a_{k j}}\right]
$$

Hence,

$$
\liminf \left(\frac{R_{n} A x}{R_{n} A y}\right) \leq 1-\lim \sup _{n \rightarrow \infty}\left[\frac{\left(\sum_{k=n}^{\infty} a_{k m}\right)}{\sum_{k=n}^{\infty} \sum_{j=0}^{\infty} a_{k j}}\right] .
$$

From (i) we get $\limsup \operatorname{su}_{n}\left[\left(\sum_{k=n}^{\infty} a_{k m}\right) / \sum_{k=n}^{\infty} \sum_{j=0}^{\infty} a_{k j}\right]=0$, and since $A$ is nonnegative matrix we have

$$
\lim _{n \rightarrow \infty}\left[\frac{\left(\sum_{k>n} a_{k m}\right)}{\sum_{k=n}^{\infty} \sum_{j=0}^{\infty} a_{k j}}\right]=0 .
$$

Hence, the proof of the theorem is complete.

In the following theorem we use another notation that was introduced by Fridy in [1] to study sequenccs that converge at different rates. Now, we use the same notation to study sequences that converge at the same rate. For sequences $x$ and $y$ that are not in $\ell^{1}$ Fridy used the partial sum $S_{N} x:=\sum_{k \leq N}\left|x_{k}\right|$ to say that $y$ diverges faster than $x$ provided that $S_{N} x=o\left(S_{N} y\right)$. Here we use $S_{N} A x \sim S_{N} A y$ to indicate that $\sum_{n \leq N}(A x)_{n}$ and $\sum_{n \leq N}(A y)_{n}$ diverge at the same rate.

THEOREM 3. If $A$ is a nonnegative matrix, then the following statements are equivalent:

(a) if $x$ and $y$ are sequences such that $x \sim y, x \in P_{0}$, and $y \in P_{\delta}$ for some $\delta>0$, then $A x$ and $A y$ are not in $\ell^{1}$ and $S A x \sim S A y$;

(b) $A$ satisfies the following conditions:

(i) $\left\{\sum_{j=0}^{\infty} a_{k j}\right\}_{k=0}^{\infty} \notin \ell^{1}$;

(ii) for each $m$,

$$
\lim _{N \rightarrow \infty}\left(\frac{\sum_{k=1}^{N} a_{k m}}{\sum_{k=1}^{N} \sum_{j=0}^{\infty} a_{k j}}\right)=0
$$


PROOF. Suppose that condition (b) is satisfied and assume that $x$ and $y$ are sequences such that $x \sim y, x \in P_{0}$, and $y \in P_{\delta}$ for some $\delta>0$. Using condition (i), it is clear that $A y$ is not in $C^{1}$. NIso, the fact that $x \sim y$ implies that for each given $\varepsilon>0$ there exists an integer $J$ such that $\left|x_{k} / y_{k}-1\right|<\varepsilon$ whenever $k \geq J$, or equivalently, if $k \geq J$ then

$$
(1-\varepsilon) y_{k} \leq x_{k} \leq(1+\varepsilon) y_{k}
$$

From this it is easy to prove that $A x$ is not in $\ell^{1}$.

Now, we need to show that $S A x \sim S A y$. Consider

$$
S_{N} A x=\sum_{k=1}^{N} \sum_{j=1}^{\infty} a_{k}, x_{j}=\sum_{k=1}^{N} \sum_{j=1}^{J-1} a_{k}, x_{j}+\sum_{k=1}^{N} \sum_{j=J}^{\infty} a_{k j} x_{j} .
$$

By using (3.4) we get

$$
\begin{gathered}
S_{N} A x \leq \sum_{k=1}^{N} \sum_{j=1}^{J-1} a_{k j} x_{j}+(1+\varepsilon) \sum_{k=1}^{N} \sum_{j=J}^{\infty} a_{k j} y_{j} \\
\leq\left(\sum_{j=1}^{J-1} x_{j}\right) \sum_{k=1}^{N}\left(\max _{0 \leq j \leq J-1} a_{k j}\right)+(1+\varepsilon) \sum_{k=1}^{N} \sum_{j=0}^{\infty} a_{k j} y_{j} \\
\leq\left(\sum_{j=1}^{J-1} x_{j}\right) \sum_{k=1}^{N}\left(\max _{0 \leq j \leq J-1} a_{k j}\right)+(1+\varepsilon) S_{N} A y .
\end{gathered}
$$

Therefore

$$
\frac{S_{N} A x}{S_{N} A y} \leq\left(\frac{\sum_{j=1}^{J-1} x_{j}}{\delta}\right)\left(\frac{\sum_{k=1}^{N}\left(\max _{0 \leq j \leq J-1} a_{k j}\right)}{\sum_{k=1}^{N} \sum_{j=0}^{\infty} a_{k j}}\right)+(1+\varepsilon) .
$$

Hence, from condition (ii) we get

$$
\lim _{N \rightarrow \infty} \frac{S_{N} A x}{S_{N} A y} \leq 1+\varepsilon,
$$

similarly, using the left-hand inequality in (3.4) we have

$$
\begin{gathered}
S_{N} A x \geq \sum_{k=1}^{N} \sum_{j=0}^{J-1} a_{k j} x_{j}+(1-\varepsilon) \sum_{k=1}^{N} \sum_{j=J}^{\infty} a_{k j} y_{j} \\
\geq\left(\sum_{k=1}\left(\min _{0 \leq j \leq J-1} a_{k j}\right)\right) \sum_{j=0}^{J-1} x_{j}+(1-\varepsilon) \sum_{k=1}^{N} \sum_{j=0}^{\infty} a_{k j} y_{j}-(1-\varepsilon) \sum_{k=1}^{N} \sum_{j=0}^{J-1} a_{k j} y_{j} .
\end{gathered}
$$

Thus

$$
\begin{aligned}
\frac{S_{N} A x}{S_{N} A y} & \geq\left(\frac{\sum_{j=0}^{J-1} x_{j}}{\sup _{j} y_{j}}\right) \frac{\sum_{k=1}^{N}\left(\max _{0 \leq j \leq J-1} a_{k j}\right)}{\sum_{k=1}^{N} \sum_{j=0}^{\infty} a_{k j}}+1-\varepsilon \\
& -\left(\frac{(1-\varepsilon) \sum_{j=0}^{J-1} y_{j}}{\delta}\right) \frac{\sum_{k=0}^{N}\left(\max _{0 \leq j \leq J-1} a_{k j}\right)}{\sum_{k=1}^{N} \sum_{j=0}^{\infty} a_{k j}} .
\end{aligned}
$$


As before, from condition (ii) we see that the first and the third terms in the previous inequality tend to zero as $N \rightarrow \infty$. Hence,

$$
\lim _{N \rightarrow \infty} \frac{S_{N} A x}{S_{N} A y} \geq 1-\epsilon
$$

From (3.5) and (3.6) we conclude that $\lim _{N \rightarrow \infty} S_{N} A x / S_{N} A y=1$, i.e., $S A x \sim S A y$.

To prove that (a) implies (b), we first need to show the necessity of condition (ii). Thus, we form two sequences $x$ and $y$ such that $x \sim y$ and both satisfy the hypothesis in (a), but $S A x$ and $S A y$ are not asymptotically equivalent if condition (ii) does not hold. Let $m$ be an arbitrary fixed positive integer and define $y_{k}:=1$ for all $k$, and

$$
x_{k}:= \begin{cases}0, & \text { if } k \leq m \\ 1, & \text { if } k>m\end{cases}
$$

It is clear that $x \sim y, x \in P_{0}$, and $y \in P_{1}$. Assume condition (ii) does not hold. For any such $m$ we have

$$
\begin{aligned}
S_{N} A x & =\sum_{k=1}^{N} \sum_{m+1}^{\infty} a_{k j}=\sum_{k=1}^{N} \sum_{j=0}^{n} a_{k j}-\sum_{k=1}^{N} \sum_{j=0}^{m} a_{k j} \\
& \leq \sum_{k=1}^{N} \sum_{j=0}^{\infty} a_{k j}-\sum_{k=1}^{N} a_{k m} .
\end{aligned}
$$

Therefore,

$$
\liminf _{N} \frac{S_{N} A x}{S_{N} A y} \leq 1-\lim \sup _{N \rightarrow \infty} \frac{\sum_{k=1}^{N} a_{k m}}{\sum_{k=1}^{N} \sum_{j=0}^{\infty} a_{k j}} .
$$

Since $A$ is a nonnegative matrix and condition (ii) does not hold, we conclude that

$$
\lim _{N \rightarrow \infty} \frac{S_{N} A x}{S_{N} A y}<1,
$$

where $S A x$ and $S A y$ are not asymptotically equivalent.

It remains to show that the sequence $\left\{\sum_{j=0}^{\infty} a_{k j}\right\}_{k=1}^{\infty}$ is not in $\ell^{1}$. Since $y$ is identically 1 , we get $(A y)_{k}=\sum_{j=0}^{\infty} a_{k j}$, and since the sequence $A y$ is not in $\ell^{1}$, we see that $\left\{\sum_{j=0}^{\infty} a_{k j}\right\}_{k=0}^{\infty}$ is not in $\ell^{1}$. Therefore the proof is complete.

In the following theorem we study the relationship between the original asymptotic equivalence that uses term by term comparison and the asymptotic equivalence that uses the remainder sum comparison.

THEOREM 4. If $x$ and $y$ are nonnegative sequences in $\ell^{1}$ such that $x \sim y$, then $R x \sim R y$.

PROOF. The assumption that $x \sim y$ implies that for a given $\varepsilon>0$ there exists an integer $N$ such that $\left|x_{n} / y_{n}-1\right| \leq \varepsilon$ for all $n \geq N$.

Hence,

$$
R_{n} x \leq(1+\varepsilon) \sum_{k \geq n} y_{k}=(1+\varepsilon) R_{n} y
$$

and,

$$
R_{n} x \geq(1-\varepsilon) \sum_{k \geq n} y_{k}=(1-\varepsilon) R_{n} y
$$

Therefore, for all $n \geq N$,

$$
(1-\varepsilon) \leq \frac{R_{n} x}{R_{n} y} \leq(1+\varepsilon),
$$

and this implies that $\lim _{n \rightarrow \infty} R_{n} x / R_{n} y=1$, i.e., $R x \sim R y$.

The next theorem shows the relationship between the original symptotic equivalence and the asymptotic equivalence that uses the partial sum comparison.

THEOREM 5. If $x$ and $y$ are nonnegative sequences not in $\ell^{1}$ such that $x \sim y$, then $S x \sim S_{y}$. 
PROOF The assumption that $x \sim y$ implies that $x_{n} / y_{n}-1=\varepsilon_{n}$, where $\lim _{n \rightarrow \infty} \varepsilon_{n}=0$. Since $x$ is nonnegative we have

$$
S_{m} x=\sum_{n \leq m}\left|x_{n}\right|=\sum_{n \leq m}\left(y_{n}+\varepsilon_{n} y_{n}\right)=\sum_{n \leq m} y_{n}+\sum_{n \leq m} \varepsilon_{n} y_{n}
$$

Therefore,

$$
\begin{aligned}
\frac{S_{m} x}{S_{m} y} & =1+\frac{\sum_{n \leq m} \varepsilon_{n} y_{n}}{\sum_{n \leq m} y_{n}}=1+\sum_{n \leq m}\left(\frac{y_{n}}{\sum_{j \leq m} y_{j}}\right) \varepsilon_{n} \\
& =1+(\bar{N} y \varepsilon)_{m},
\end{aligned}
$$

where $\bar{N} y$ is the Nörland-type matrix $\left[3\right.$, p. 45]. Since $y$ is not in $\ell^{1}, \bar{N}_{y}$ is regular; therefore $\lim _{m \rightarrow \infty}(\bar{N} y \varepsilon)_{m}=0$. Thus we have $\lim _{m \rightarrow \infty} S_{m} x / S_{m} y=1$, i.e., $S x \sim S y$.

In the last result we study the relationship between the original asymptotic equivalence and the asymptotic equivalence that compares the "supremum of the remaining terms."

TIIEOREM 6 . If $x$ and $y$ arc nonvanishing null sequences such that $x \sim y$, then $\mu x \sim \mu y$.

PROOF. The hypothesis $x \sim y$ implies that for $\varepsilon>0$ there exists an integer $N$ such that if $k \geq N$ then

$$
(1-\varepsilon) y_{k} \leq x_{k} \leq(1+\varepsilon) y_{k}
$$

For $n \geq N$ we have

$$
\mu_{n} x=\sup _{k \geq n}\left|x_{k}\right| \leq(1+\varepsilon) \sup _{k \geq n}\left|y_{k}\right|
$$

Hence,

$$
\lim \sup _{n} \frac{\mu_{n} x}{\mu_{n} y} \leq 1+\varepsilon
$$

Also,

$$
\mu_{n} x=\sup _{k \geq n}\left|x_{k}\right| \geq(1-\varepsilon) \sup _{k \geq n}\left|y_{k}\right|
$$

Hence,

$$
\liminf \frac{\mu_{n} x}{\mu_{n} y} \geq 1-\varepsilon .
$$

Therefore we conclude that $\lim _{n \rightarrow \infty} \mu_{n} x / \mu_{n} y=1$, i.e., $\mu x \sim \mu y$.

REMARK. The converse of Theorems 5 and 6 is not true i.e., there exist sequences $x$ and $y$ such that $S x \sim S y$ and $\mu x \sim \mu y$ but $x$ is not asymptotically equivalent to $y$. It is cnough here to give the two sequences $x$ and $y$ and leave the proof to the reader.

$$
\begin{aligned}
& x_{n}:=\left\{\begin{array}{cl}
\frac{2}{n+1} & , \text { if } n \text { is odd, } \\
\frac{1}{n} & , \text { if } n \text { is even. }
\end{array}\right. \\
& y_{n}:=\left\{\begin{array}{cl}
\frac{1}{n+1} & , \text { if } n \text { is odd } \\
\frac{2}{n} & , \text { if } n \text { is even. }
\end{array}\right.
\end{aligned}
$$

ACKNOWLEDGMENT. This work is a portion of the author's doctoral dissertation, written under the supervision of Professor J.A. Fridy at Kent State University. 


\section{REFERENCES}

1. Fridy, J.A. Minimal Rates of summability, Can. J. Math., Vol. XXX, 4(1978), 80S-816.

2. Pobyvancts, I.P. Asymptotic Equivalence of some Linear Transformation Defined by a Nonnegative Matıix and Reduced to Generalized Equivalence in the sense of Cesàro and Abel, Mat. Fiz. 28(1980), 8.3-87, 123.

3. Powel, R.E. and Shah, S.M. Summability Theory and its Applications, Van Nostrand Rienhold, London, 1972. 


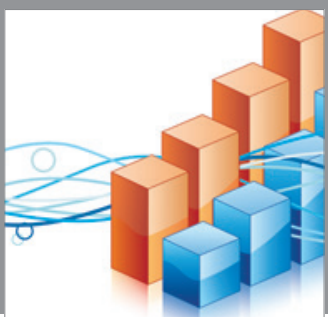

Advances in

Operations Research

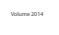

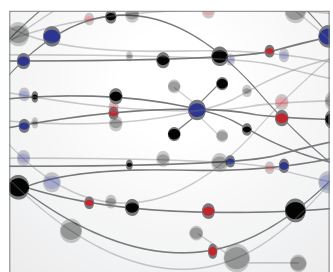

\section{The Scientific} World Journal
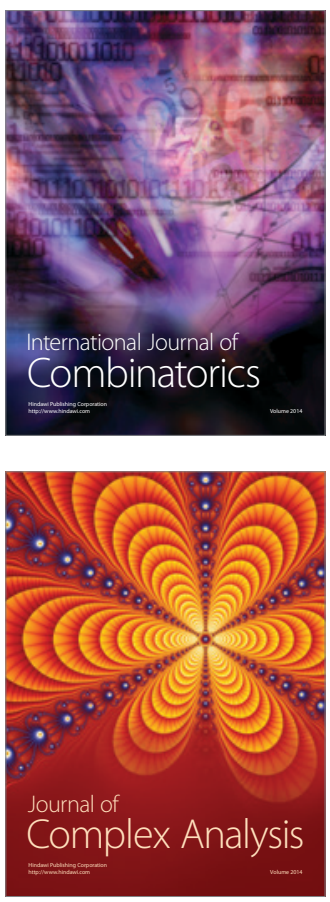

International Journal of

Mathematics and

Mathematical

Sciences
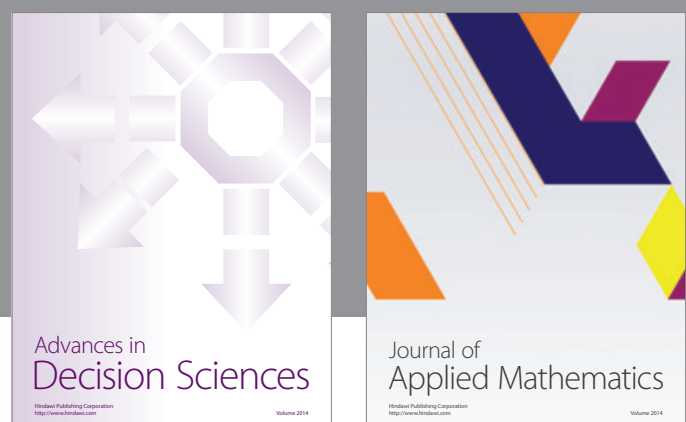

Journal of

Applied Mathematics
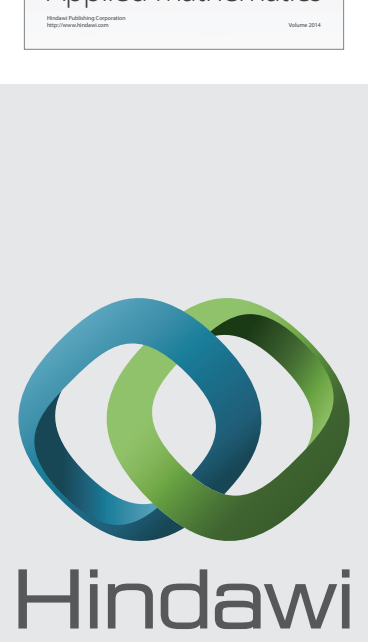

Submit your manuscripts at http://www.hindawi.com
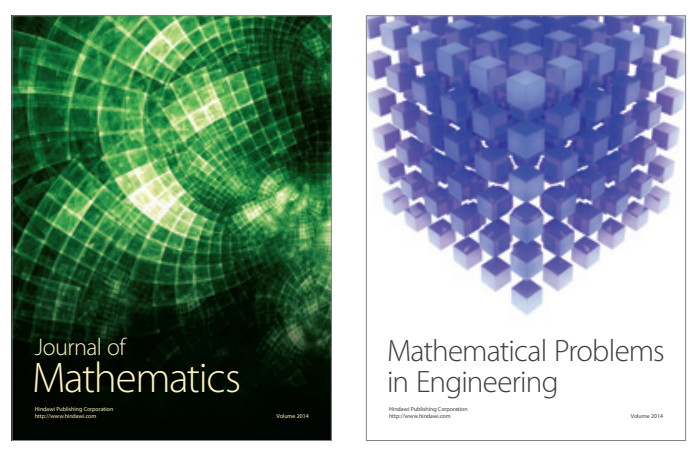

Mathematical Problems in Engineering
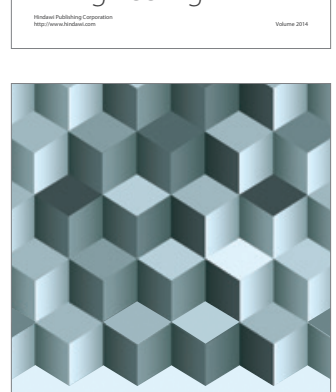

Journal of

Function Spaces
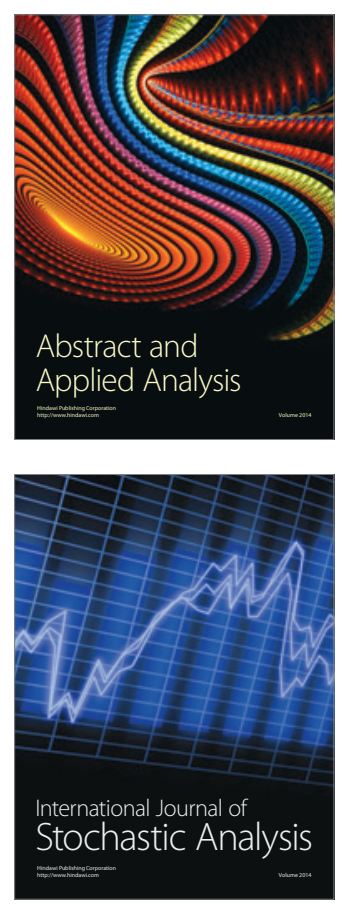

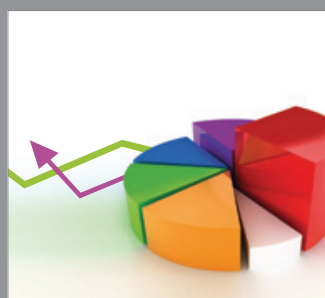

ournal of

Probability and Statistics

Promensencen
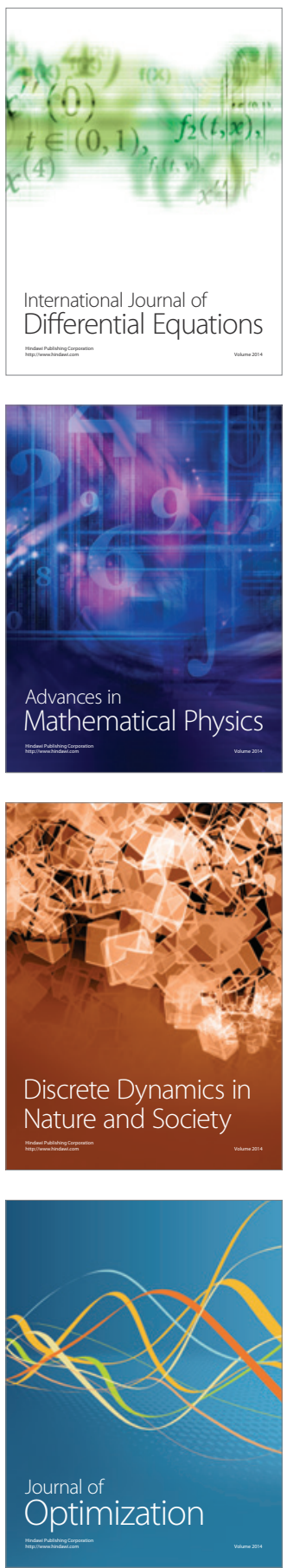